\title{
Gault Millau für Spitäler und Heime: vom Benchmarking und «Benchmarketing»*
}

Dr. rer. pol. Gustav Egli, PD Dr. med. Julian Schilling, Armin Dörzbach

* Résumé en français voir p. 311

Korrespondenz:

Residenz Bethesda Küsnacht Rietstrasse 25

CH-8700 Küsnacht

Tel. 0449132702

bethesda.kuesnacht@bluewin.ch

\section{Zusammenfassung}

Benchmarking heisst, Produkte und Leistungen mit anderen Betrieben zu vergleichen, aus den Vergleichen mit den Besten zu lernen und, wo notwendig, Verbesserungen zu realisieren.

Eine Diplomarbeit am BWI ETH hat dazu ein vergleichsweise kostengünstiges und internetfähiges Messsystem entwickelt und bei Pilotheimen in der Region Zürich getestet. Die Zeit für einen «Gault Millau» ist aber noch nicht reif. Als nächste Phase folgt die Validierung des Messsystems. Dabei stehen die wissenschaftlichen Anforderungen an Messungen wie Validität (messen die verschiedenen Einzelindikatoren, tatsächlich, was sie zu messen versprechen), die Realibilität (Reproduzierbarkeit), die Relevanz (sind die einzelnen Indikatoren für die primären Kunden von Bedeutung) und die Vergleichbarkeit (wird Gleiches mit Gleichem verglichen) im Fokus. Zur Beantwortung der Fragestellungen werden in $\mathrm{Zu}$ kunft weitere Daten erhoben. Bei einer genügenden Menge Datensätze können zu einem späteren Zeitpunkt nicht nur eine Bewertung des Systems mit Verbesserungsvorschlägen, sondern auch ein «Rating» vorgenommen werden. Ob sich das Messsystem für internes oder öffentliches Benchmarking eignet und ob die einzelnen Betriebe mit den Daten «Benchmarketing» (beziehungsweise Werbung mit Daten, wo man am besten abschneidet) betreiben können, wird erst die Zukunft zeigen.

\section{Hintergrund}

Betriebe wie Spitäler und Heime sind menschlich sensible Orte, wo Patienten, Bewohner und andere Kunden Könige sein sollen und zum Teil auch sein wollen. Heute, wo viele ältere Menschen nach dem Alter von 80 in Altersheime eintreten, haben die meisten Heime auch Pflegeabteilungen eingerichtet. Es besteht ein Trend weg von traditionellen Altersheimen Richtung Pflegeheim und Seniorenresidenzen mit altersgerechten kleineren Wohnungen [1].

Für das vielfältige Angebot an Gesundheitseinrichtungen gibt es heute keinen «Gault Millau». Es gibt auch keine schweizweit anerkannten Messgrössen, welche einheitlich gemessen und somit von den Kunden konsultiert werden können. Die Wahl betreffend «Güte» einer Einrichtung fällt somit vielen Kunden schwer. Fast unmöglich ist es für alte, zum Teil verwirrte Menschen, die seit längerem in einem Heim sind, die Pflege- und Betreuungsqualität zu vergleichen und zu beurteilen. Auch ein Verantwortlicher eines Betriebes der Gesundheitsversorgung hat kaum schlüssige Kennziffern, seien sie harter oder weicher Natur, um seinen Betrieb mit anderen Betrieben vergleichen zu können. Das Thema ist allerdings nicht ganz neu. Systeme, welche auch in der Lage sind, qualitative Aussagen zu Ergebnissen von Leistungen bereitzustellen, wurden schon verschiedentlich übernommen, entwickelt und weiterentwickelt $[2,3]$. Die bekannten Administrationssysteme wie beispielsweise das «Resident Assessment Instrument» (RAI) oder BESA vermögen Anhaltspunkte zur Qualität der Leistungen der Institutionen der Langzeitpflege zum Teil auf Knopfdruck zu generieren [4]. Die Systeme sind jedoch primär zur Leistungserfassung, Administration und Verrechnung entwickelt worden [5]. Die Aussagen zur Qualität der Leistungen beschränken sich somit naturgemäss auf Teilaspekte der Betreuung betagter Menschen [5]. Zudem besteht die Limitierung, welche alle Selbstmessungen aufweisen: Die Direktbetreuenden beurteilen mittels Kriterien mit grossen Graubereichen ihre Arbeit selber. Ein «Gault Millau» ist aus den Messungen aufgrund der obengenannten Anforderungskriterien Validität, Realibilität, Relevanz und Vergleichbarkeit zum heutigen Zeitpunkt noch nicht gegeben.

Qualitätsmanagement und Benchmarking Sehr wohl gibt es sogenannte Qualitätsmanagementsysteme, welche einer Institution Anleitungen geben können, ihre gelebte Qualität im Haus zu überprüfen und wo notwendig anzupassen [6]. Europäische Beispiele sind: European Foundation for Quality Management (EFQM) oder Industrial Standard Organisation (ISO). Für Schweizer Heime gibt es davon abgeleitet zum Beispiel «Qualität als Prozess» (QAP). Wenn ein Betrieb EFQM, ISO oder QAP anwendet, weiss er deswegen allerdings noch nicht, wo er bezüglich Qualität der Leistungen im Vergleich zu anderen Betrieben steht. 
Die Wirtschaft kennt vergleichende Systeme oder Benchmarkingsysteme (BMS) seit langem. Üblicherweise setzt irgendein Betrieb mit seinen Produkten oder Leistungen sogenannte Standards. Standards sind in diesem Kontext nicht als Handlungsanweisungen, sondern als zu erreichende Leistungsergebnisse zu verstehen [7]. In einem BMS werden sogenannte Bestmarkierungen erfasst und mit den Standards oder «Marks» von anderen Firmen verglichen. Jeder kann sich somit an den Standards der Besten messen, denn «Gut ist nicht was besser sein könnte». Diese Einsicht kann ein Betrieb aber nur erhalten, wenn er sich selber verpflichtet, sich mit anderen, möglichst den Besten seiner Branche zu messen. Würden viele Betriebe sich an einem BMS beteiligen, könnte sich in Zukunft vielleicht zeigen, dass Betriebe, die ein bestimmtes Qualitätsmanagement verwenden, hohe Standards erreichen. So gesehen könnte ein BMS ein vergleichendes Messsystem für Qualitätsmanagementsysteme sein.

Dies hat die Wirtschaft seit langem erkannt um mittels Benchmarking die Qualität und damit die Konkurrenzfähigkeit des Unternehmens zu sichern. Nicht so im Gesundheitswesen: Weder im Langzeitbereich noch im Akutbereich wird mit validen, reliablen und relevanten Benchmarksystemen gearbeitet. Dies wäre allerdings gesetzlich gefordert (KVG Art. 58) [8]. Daraus resultieren könnten eine verbesserte Betriebstransparenz und Effizienz, mit dem Ziel der Qualitäts- und Kostenstrukturverbesserung.

\section{HeBes}

Am BWI der ETH Zürich wurde dazu eine Diplomarbeit verfasst [9] und ein dazugehöriges BMS entwickelt: «Heimbenchmarkingsystem Schweiz» (HeBeS). HeBeS wurde an den Institutionen Bethesda-Küsnacht, Pflegheim am See - Küsnacht, Zumipark - Zumikon und Alterszentrum Burgdorf getestet. Dem System liegt ein anspruchsvolles Ziel zugrunde: verstummten Patienten und Bewohnern wieder eine Art «Stimme» $\mathrm{zu}$ geben. Bildlich gesprochen wird mit über 120 Qualitätsindikatoren innerhalb eines Betriebes in der Pflege, bei den Therapien, bei den ärztlichen Diensten, in der Hotellerie, in der Verwaltung usw. die «Temperatur» gemessen. Die Qualitätsindikatoren erfassen nicht nur den Struktur- und Prozessbereich, sondern auch Kosten und Ergebnisdaten wie beispielsweise Infekte oder Stürze. Ist die Temperatur einzelner Bereiche innerhalb eines Betriebes beispielsweise 36 Grad, von wenigen optimale 37 Grad und von einigen Bereichen 38 Grad, könnten jene Be-

\section{Points Gault et Millau pour}

\section{les hôpitaux et les homes:}

\section{du benchmarking au «bench-}

\section{marketing»}

Pratiquer le benchmarking, terme que l'on pourrait traduire par «étalonnage», consiste à comparer ses produits et ses prestations avec ceux de différentes entreprises, à en tirer les leçons qui s'imposent et à procéder aux améliorations nécessaires en s'inspirant des meilleurs.

Un travail de diplôme effectué à l'institut d'économie d'entreprise (BWI) de I'EPFZ a permis de développer un système de mesure des prestations relativement peu coûteux, utilisable sur internet, et de le tester dans des homes-pilotes de la région de Zurich. Les temps ne sont toutefois pas encore mûrs pour établir un classement de type «Gault et Millau». L'étape suivante consistera à valider ce système de mesure. Pour ce faire, il faudra notamment examiner s'il remplit les exigences suivantes en matière de mesures scientifiques: validité (les différents indicateurs individuels mesurent-ils effectivement ce qu'ils sont censés mesurer?), compatibilité avec la réalité (reproductibilité), pertinence (les indicateurs individuels sont-ils significatifs pour les clients primaires?) ainsi que comparabilité (compare-t-on ce qui est véritablement comparable?). Pour répondre aux questions qui découleront de ce bilan, de nouvelles données seront ensuite prélevées. Lorsque volume des données disponibles sera suffisant, I'on pourra procéder à une évaluation du système débouchant sur des améliorations ainsi qu'à une classification («rating»). Seul I'avenir permettra de savoir si ce système de mesure est approprié pour effectuer un benchmarking interne ou public et si les établissements pourront utiliser les données obtenues pour leur «benchmarketing». Par ce dernier terme, on entend I'utilisation à des fins publicitaires des données et domaines où l'on obtient les meilleurs résultats. 
reiche mit einer Untertemperatur von 36 oder einer Übertemperatur von 38 Grad möglicherweise einiges bei Betrieben mit Bereichen mit optimalen 37 Grad lernen.

HeBeS erfasst in der Praxis 120 harte und weiche Messkriterien oder Fingerprints von einem Betrieb. Diese können sein: Wer ist der Träger des Betriebes, wie gross ist die durchschnittliche Zimmergrösse von einem Ein-Bettoder Zwei-Bett-Zimmer, haben alle Zimmer Lavabo, Dusche und WC, wie gross ist die Anzahl Menüs beim Mittagessen, gibt es eine hausinterne Kapelle mit eigener Seelsorge, wie viele Psychopharmaka werden an einem Stichtag den Bewohnern verabreicht, wie viele Harninfekte (mit und ohne Katheterisierung) kommen vor, wie viele Personen leiden an Harninkontinenz, wie viele Stürze (mit und ohne Folgen) kommen vor, ist die Patientenverfügung institutionalisiert, welches Angebot mit Aktivierungsspezialistinnen steht den Bewohnern zur Verfügung, wie viele Pflegende und wieviel diplomiertes Fachpersonal gibt es pro Bewohner, wie hoch ist deren Fluktuation, gibt es hausintern ein Restaurant, eine Cafeteria, einen Coiffeur, deren Öffnungszeiten in Stunden, wie hoch ist der Gebäudeversicherungswert des Gebäudes, wann wurde das Gebäude erbaut, wie hoch ist der Betriebsergebnisüberschuss oder die Kostenunterdeckung usw.?

Diese Messkriterien werden anonym in eine Internetplattform www.hebes.ch eingegeben. HeBeS errechnet aus den Messkriterien automatisch die vordefinierten Kennziffern und wertet diese statistisch aus. Die resultierenden Kennziffern eines Betriebes werden den durchschnittlichen Kennziffern, den Richtwerten von Experten und den besten Betrieben gegenübergestellt. Der Verantwortliche eines Betriebes kann aus diesen Vergleichen Rückschlüsse über die Art und Weise seines Betriebsgeschehens ziehen. In Geschäftsleitungen und den entsprechenden zuständigen Arbeitsgruppen können die Kennziffervergleiche interpretiert werden. Dies mit dem Ziel, entsprechende «Bestmarkierungen» erreichen zu können. Das System kann somit helfen, einen Betrieb mit anderen Betrieben zu vergleichen. Die Vorteile gegenüber heute verwendeten Vergleichssystemen sind der Preis: Fr. 100.- während der Pilotphase pro vergleichende Auswertung, später sind Fr. 999.- geplant, die einfache elektronische und zeitsparende Datenerfassung und der Fokus auf ältere, verwirrte Patienten oder Bewohner, welche nicht mehr befragt werden können.

\section{Interpretation}

Mit diesem Messsystem wurde zum Teil Neuland betreten, aber noch kein Gault Millau geschaffen. Das Werkzeug für Betriebsvergleiche über weiche und zum Teil harte Betriebsdaten, von dem jeder Betriebsverantwortliche etwas gewinnen kann, liegt nach einer Piloterhebung vor. Aufgrund der noch zu geringen Datenmenge kann jedoch zum heutigen Zeitpunkt zur Validität und Realibilität noch keine Aussage gemacht werden. Die Relevanz darf vorsichtig beurteilt als gegeben betrachtet werden und die Vergleichbarkeit ist gegeben. Ob sich aus den Daten und Vergleichen zukünftig ein Gault Millau entwickelt und ob ein solcher für Pflegeund Betreuungseinrichtungen Sinn macht, wird die Zukunft zeigen [10].

Literatur

1 Saup W. [Home life of the aged - psychological aspects]. Z Gerontol 1986;19(5):342-7.

2 Brazil K, Royle JA, Montemuro M, Blythe J, Church A. Moving to evidence-based practice in long-term care: the role of a Best Practise Resource Centre in two long-term care settings. J Gerontol Nurs 2004;30(3):14-9.

3 Bryant LL, Floersch N, Richard AA, Schlenker RE. Measuring healthcare outcomes to improve quality of care across post-acute care provider settings. J Nurs Care Qual 2004;19(4):368-76.

4 Habermann R, Butts N, Powers J, Pichert JW. Physician behavior changes following CME on the prospective payment system in long-term care: a pilot study. J Am Med Dir Assoc 2002; 3(1):12-5.

5 Bjorkgren MA, Fries BE, Hakkinen U, Brommels M. Case-mix adjustment and efficiency measurement. Scand J Public Health 2004;32(6):464-71.

6 Selbmann HK. [Assessment and certification of hospital care in Germany]. Bundesgesundheitsblatt Gesundheitsforschung Gesundheitsschutz 2004;47(2):103-10.

7 Schilling J. Qualitätssicherung in der Medizin: Methodik zur Indikationsforschung in der interventionellen Kardiologie und in der Gynäkologie. (Quality Assurance in Medicine: Methodology for research on indications in interventional cardiology and gynaecology). Frankfurt am Main: pmi Verlagsgruppe GmbH; 1998.

8 Bundesgesetz über die Krankenversicherung (KVG). 1994:38.

9 Dörzbach A. Kennzahlen-System zur Unterstützung der Qualitätsbeurteilung in Pflegeheimen. Nachdiplomarbeit am BWI - ETH. Aarau: HPlus; 2002.

10 Werner RM, Asch DA. The unintended consequences of publicly reporting quality information. JAMA 2005;293(10):1239-44. 